Supporting information for

\title{
Constructing Oriented Two-Dimensional Large-Sized Modified Graphene Oxide Barrier Walls in Brominated Butyl Rubber to Achieve Excellent Gas Barrier Properties
}

\author{
Siqiang Yang, ${ }^{a}$ Hong $W u,{ }^{a, b *}$ Chunhai Li, ${ }^{a *}$ Ying Xiong, ${ }^{a}$ Shaoyun Guo ${ }^{a}$
}

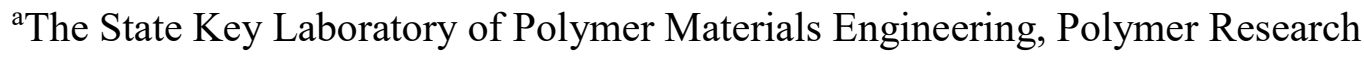
Institute, Sichuan University, Chengdu 610065, China

${ }^{b}$ Research Center for Application of Graphene (Sichuan University-WuXi), Wuxi 214174, China

\section{Corresponding Authors}

*E-mail: wh@scu.edu.cn. Fax: 86-028-85466077 (H.W.).

*E-mail: 1polymer@scu.edu.cn. Fax: 86-028-85466077 (C.L.).

1. Structural comparison diagram of oriented 2D large-sized mGO barrier walls and the segregated structure.

In general, the most common way to achieve the selective distribution of GO is constructing segregated structures, as shown in Figure S1b. However, the oriented 2D large-sized mGO barrier walls are constructed in this work, as shown in Figure S1a. Compared with the common segregated structures, our work has the following advantages: 
(1) The oriented 2D large-sized mGO barrier walls with their in-plane direction perpendicular to the diffusing direction of the permeable gas molecules, are more efficient in extending the diffusing path of the gas molecules, and thus are more effective in enhancing the gas barrier properties;

(2) the 2D large-sized mGO barrier walls are orderly distributed in the continuous BIIR matrix, indicating that the mechanical defects associated with the brittle inorganic $\mathrm{mGO}$ as the continuous phase in the segregated structure could be overcome, as shown in Figure S1b.

(a)

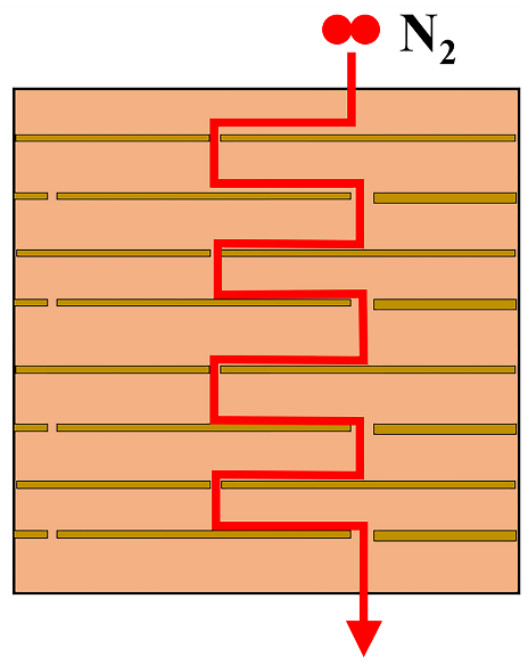

Oriented 2D large-sized mGO barrier walls Our work (b)

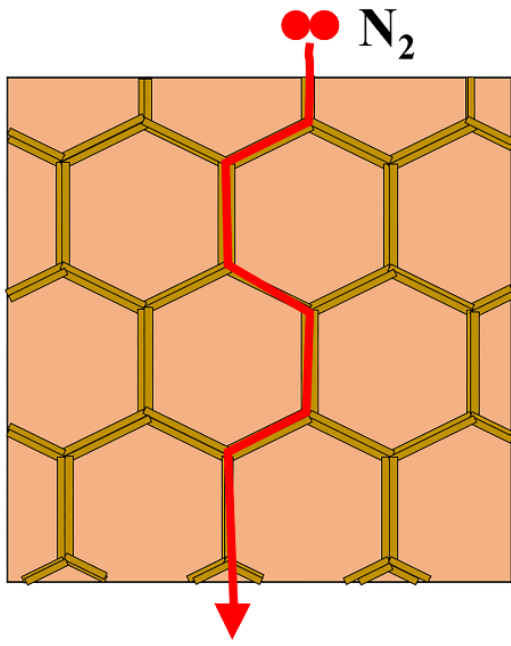

Segregated structure

\section{Other methods}

Figure S1. Structural comparison diagram of (a) oriented 2D large-sized mGO barrier walls and the segregated structure. 
2. Thermogravimetric analysis of GO and GO-OTAB.

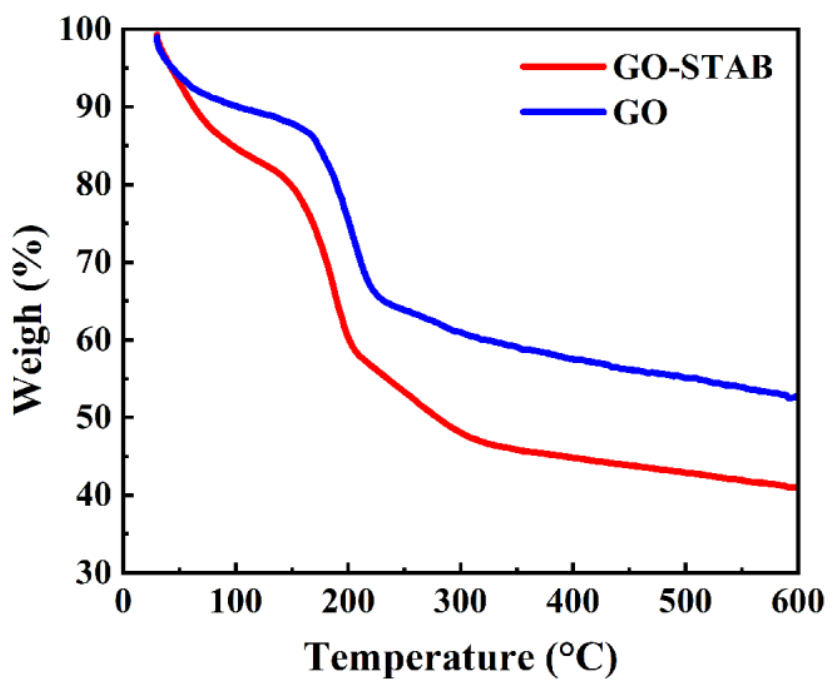

Figure S2 TG curves of GO and GO-OTAB. 


\section{The detailed information of the characteristic peaks of GO}

Table S1 The characteristic peak of GO and its attribution.

\begin{tabular}{|c|c|c|c|c|}
\hline \multirow{2}{*}{$\begin{array}{l}\text { Peak } \\
\text { location }\end{array}$} & \multicolumn{4}{|c|}{ Attribution } \\
\hline & $\begin{array}{l}\text { Vibration } \\
\text { mode }\end{array}$ & Bond & Functional group & $\begin{array}{c}\text { Functional group } \\
\text { position }\end{array}$ \\
\hline $3430 \mathrm{~cm}^{-1}$ & stretching & $\mathrm{O}-\mathrm{H}$ & hydroxyl & the surface of GO \\
\hline $1630 \mathrm{~cm}^{-1}$ & bending & $\mathrm{O}-\mathrm{H}$ & hydroxyl & the surface of GO \\
\hline $1730 \mathrm{~cm}^{-1}$ & stretching & $\mathrm{C}=\mathrm{O}$ & $\begin{array}{l}\text { carbonyl or } \\
\text { carboxyl }\end{array}$ & the edge of GO \\
\hline $1225 \mathrm{~cm}^{-1}$ & stretching & $\mathrm{C}-\mathrm{OH}$ & carboxyl group & the edge of GO \\
\hline $1053 \mathrm{~cm}^{-1}$ & stretching & $\mathrm{C}-\mathrm{O}-\mathrm{C}$ & epoxy group & the surface of GO \\
\hline
\end{tabular}

\section{The measurement method of sublimation rate of cyclohexane and water}

Two Petri dishes of the same diameter were labeled as A and B, respectively, and the mass of both was weighed. To the Petri dish A, $100 \mathrm{ml}$ of cyclohexane (CHY) was added while $60 \mathrm{ml}$ of water was added to the Petri dish B. After weighing the mass again, the culture dishes A and B were freeze-dried in a freeze-dryer. During freezedrying, the masses of Petri dishes A and B were weighed at intervals of 0.5 hours until they were completely removed. Organize relevant data to make a mass loss rate of $\mathrm{CHY}$ 
and water - time curve.

5. SEM image of the cross-section of the freeze-dried sample

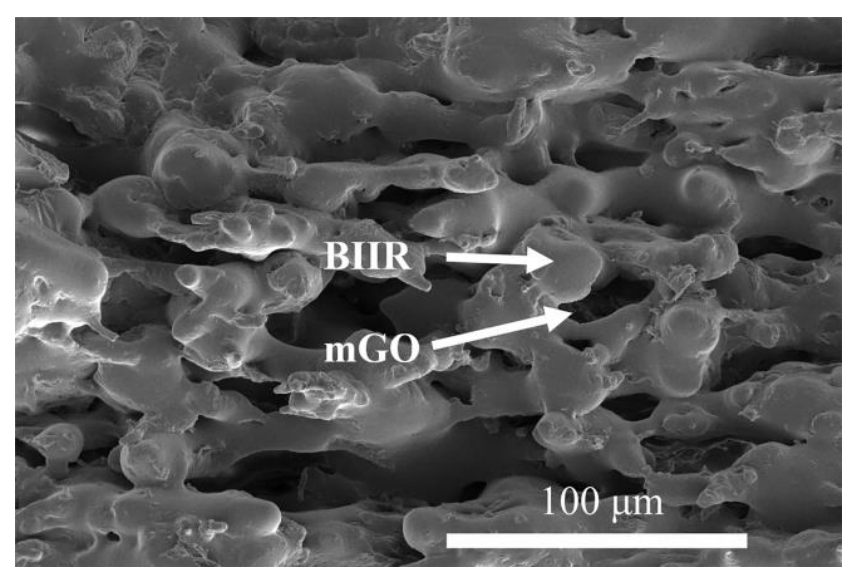

Figure S3 SEM image of the cross-section of the freeze-dried sample. 